\title{
THE TAIL INDEX OF EXCHANGE RATE RETURNS
}

\author{
Kees G. KOEDIJK \\ Erasmus University Rotterdam, 3000 DR Rotterdam, The Netherlands \\ Marcia M.A. SCHAFGANS \\ World Bank, Washington, DC 20433, USA \\ Casper G. de VRIES* \\ K.U. Leuven, 3000 Leuven, Belgium
}

Received May 1988, revised version received January 1990

In the literature on the empirical distribution of foreign exchange rates there is now consensus that exchange rate yields are fat-tailed. Three problems, however, persist: (1) Which class of distribution functions is most appropriate? (2) Are the parameters of the distribution invariant over subperiods? (3) What are the effects of aggregation over time on the distribution? In this paper we employ extreme value theory to shed new light on these questions. We apply the theoretical results to EMS data.

\section{Introduction}

In a recent issue of this Journal, Boothe and Glassman (1987) provided a comprehensive study and exhaustive summary of the theoretical and empirical work on the unconditional distribution of foreign exchange rate returns. Taking their excellent survey as our point of departure, it appears that three important questions are unresolved. ${ }^{1}$ While the profession agrees on the stylized fact that shorter term foreign currency returns are fat-tailed, the three questions center on the precise implications of this tail-fatness for the

\footnotetext{
*We wish to thank Laurens de Haan for his invaluable theoretical insights. Guido Imbens, Peter Schotman, an anonymous referee and the editors of this journal provided valuable comments. leko Sevinga provided able research assistance. We profited from presentations given at Brown University, the University of Zürich and the European Economic Association meetings 1988. The major part of this work was conducted while all three authors were at Erasmus University Rotterdam.

'The survey by Boothe and Glassman gives the state of the art in modelling the exchange rate yield distribution. Therefore we deem it unnecessary to provide an exegesis of the existing literature. Rather, for each issue we discuss, we refer to the corresponding discussion in Boothe and Glassman. Thus, the reader should bear in mind that this is not a critique, but a continuation. Our approach, though, is quite novel.
}

0022-1996/90/\$03.50 (C) 1990-Elsevier Science Publishers B.V. (North-Holland) 
distribution function. ${ }^{2}$ The questions are directly related to the exact amount of tail-fatness that is involved. In this paper we suggest ways to resolve some of those issues by methods that rely heavily on the presumption of fat tails, but are robust against the specific amount of leptokurtosis that is present. We discuss the three issues in turn.

First, several non-nested distributions have been suggested as the appropriate yield data generating functions, each varying in their degree of tailfatness. At the one end of the spectrum we find the normal, which can be readily dismissed due to the excessive amount of outliers in the data. Closely related are the mixtures of the normal distribution, which are not appropriate for the same reason. The Student- $t$ class with finite variance takes an intermediate position. The Student- $t$ has been suggested, because it combines a finite variance with fat tails. A finite variance is often presumed in economic theory, and fat tails are an empirical regularity. For this class some moments do exist, but not all. At the other end of the spectrum are the sumstable laws. The main attraction of the sum-stable laws is the additivity property together with the fat tails property. As the existence of moments is directly related to the amount of probability mass in the tails, the question about the appropriate class of distributions is directly related to the amount of tail-fatness that exists in the data.

The problem is that these alternative models are non-nested, so that estimates are necessarily dependent on the maintained hypothesis. Therefore, the competing hypotheses cannot be directly tested against each other, i.e. likelihood ratios cannot be used. The Cox procedure is not applicable either, as the second moment may not exist [c.f. White (1982)]. Boothe and Glassman (1987) suggested employing the chi-square goodness of fit test, but, as they indicate, it has the drawback that one must break up the empirical distribution function into arbitrary intervals. Moreover, estimates from different samples cannot be compared in this way. As it turns out, the tail behavior of the alternatives can be parameterized by the so-called tail index $\alpha$ from the limit law of the distribution of the maxima. In case of a Student- $t$, $\alpha$ equals the degrees of freedom, and for the non-normal sum-stable law $\alpha$ equals the characteristic exponent. The idea is to focus on the tail behavior in order to recover $\alpha$. Below, we present ways in which the tail index can be estimated directly, i.e. in such a way that the different hypotheses appear as nested alternatives. The advantage is that estimates do not rely on one of the alternatives as a maintained hypothesis.

The estimation procedure also implies an asymptotic confidence interval for the estimate $\hat{\alpha}$. This gives the present method a clear advantage over traditional procedures like Fama and Roll's (1971) method. As the Student-t

\footnotetext{
${ }^{2}$ In this paper the term tail-fatness refers to the probability weight in the tails of the distribution and is defined in section 2. By fat-tailed distributions we mean distributions that have fatter tails than the normal distribution.
} 
class with finite variance requires $x>2$, and the leptokurtic sum-stable alternative has $\alpha<2$, the confidence interval may be used to directly discriminate between these alternatives.

Second, it is sometimes felt desirable to test for the stability of the parameters over different subsamples, i.e. related to a division of time or different exchange rates. For example, Boothe and Glassman divided their sample in halves to detect structural breaks, and compared the two subperiod distribution estimates on the basis of the non-parametric median quartile test. Here we will not test jointly for the stability of all parameters, but only for the tail index. It should be noted that the latter may be subsample invariant, while equality of the entire distribution is rejected.

Analysis of the stability of $\alpha$ is especially relevant for the case of the EMS. One of the main objectives of the EMS was to delimit extreme swings in the bilateral exchange rates. The tail index $\alpha$, by its very nature, is a suitable measure of the extremal behavior of exchange rate movements. Hence, one may wonder whether the formation of the EMS has been conducive to changing $\alpha$. Accordingly, we will gauge the effects of the EMS formation by employing the stability test. Sometimes, the possible variation in parameters is modelled explicitly. For example, the ARCH process, introduced by Engle (1982), conditions the current variance on past realizations, thereby generating clusters of high and low volatility. The interesting economic question is then whether the fluctuation margins have affected this kind of volatility clustering. It is outside the scope of this paper to do full justice to these processes, but we do address the issue of dependent data to some extent.

Third, there is the question of the effect of aggregation over time on the distribution of exchange rate returns. As is well known from economics principles, returns are additive over time. For this reason it is often felt desirable to preserve this additivity into the distribution of the returns. As it turns out, the class of sum-stable distributions is the only one that possesses this property. The combination of fat tails and the additivity property makes this class appealing. The Student- $t$ class has been advocated mainly because of its finite variance while still being leptokurtic. The price is the loss of additivity, although - and this seems to have gone unnoticed in the economics literature - additivity in the tails of the Student- $t$ is preserved. Thus, the sums of Student- $t$ variates have the same tail index as thcir summands. Hence, the requirement of additivity is less restrictive than it appears at first sight.

Additivity has been tested for by estimating the parameters of the sumstable distribution on high and low frequency data, and comparing such estimates. A tendency towards 2 for the characteristic exponent has sometimes been observed for the lower frequency data. Although formal testing for additivity is difficult due to the interdependency between the high and low frequency data, we go some way towards alleviating this problem. See 
Diebold (1988a) for a related discussion. Especially the reduction in efficiency due to the use of low frequency data will become apparent.

Given the dispute over the specific distribution, and the fact that returns are fat-tailed, we suggest employing extreme value theory that is robust against the former and exploits the latter observation. ${ }^{3}$ This theory focuses on the limit distribution of maxima, in analogy with the central limit theorem for sums. As the distribution of the maximum is especially sensitive to the tail behavior, the limit laws exhibit a direct relationship with the amount of tail-fatness. In fact, the tail index $\alpha$ characterizes the limit law. Below we formulate the above models as nested hypotheses about $\alpha$, and estimate $\alpha$ directly. This procedure shares the advantage with the central limit theorem in that no specific assumptions about the data generating mechanism have to be made. It allows for asymptotic inferences about the main parameter of concern. This seems to accord well with Boothe and Glassman's (1987, p. 314) remark that not 'one of the alternatives is the distribution for exchange rate changes'. In other words, for many questions in the area of exchange rates it is sufficient to know some of the properties of the distribution function $F$, such as its tail index, without knowing $F$ in detail. Thus, our methodology is not to try to recover the entire distribution that generates the returns, but only the tail of the distribution. This is not to say that changes in the non-extreme parts are of no interest; however, by giving up this information the tail analysis gains in robustness.

The next section provides the necessary theoretical background. In section 3 we apply the theory to EMS currency data and the British pound.

\section{The theory of extremes}

Consider a stationary sequence $X_{1}, X_{2}, \ldots$ of independent and identically distributed (i.i.d.) random variables with a distribution function $F$. Suppose one is interested in the probability that the maximum

$$
M_{n}=\max \left(X_{1}, X_{2}, \ldots, X_{n}\right)
$$

of the first $n$ random variables is below a certain level $x$. As is well known, this probability is given by:

$$
P\left\{M_{n} \leqq x\right\}=F^{n}(x) .
$$

Extreme value theory studies the limiting distribution of the order statistic $M_{n}$ appropriately scaled. That is, one is interested in the conditions under which there exist suitable normalizing constants $a_{n}>0$, and $b_{n}$ such that

\footnotetext{
${ }^{3}$ This approach may also prove useful in theoretical economics. For example, Flood and Garber (1984) use the limit law for extremes in their analysis of the peso problem. McFadden (1973) constitutes an example from the area of qualitative response models.
} 


$$
P\left\{a_{n}\left(M_{n}-b_{n}\right) \leqq x\right\} \stackrel{\sim}{\rightarrow} G(x),
$$

i.e.

$$
F^{n}\left(x / a_{n}+b_{n}\right) \stackrel{\leftrightarrow}{\rightarrow} G(x),
$$

where $G(x)$ is one of the three asymptotic distributions that are defined below, and $w$ stands for weak convergence. If (3) holds, we shall say that $F$ belongs to the domain of attraction of $G$, and write $F \in D(G)$.

Define the class of limiting laws which may appear in (3) as follows:

Definition 1. A non-degenerate distribution function (d.f.) $G$ is called maxstable if there exist real constants $A_{n}>0$ and $B_{n}$ such that for all real $x$ and $n=1,2, \ldots$

$$
G^{n}\left(A_{n} x+B_{n}\right)=G(x) .
$$

One can show that if (3) holds, then $G$ is max-stable. The main result is the Extremal Types Theorem:

Theorem 1. The max-stable distributions can be represented by

$$
G_{y}(x)=\exp \left(-[1+\gamma x]^{-1 / \gamma}\right),
$$

for values of $x$ such that $1+\gamma x \geqq 0$.

Note that the limit law is characterized by the parameter $\gamma$ which is the inverse of the tail index $\alpha$ referred to in the introduction. See Mood et al. $(1974$, p. 261) for an introductory account of this result. Leadbetter et al. (1983) provide a comprehensive treatment, with relatively straightforward proofs. Chapter 3 of the latter reference also contains an extensive treatment of dependent sequences. The dependency affects the norming constants but not the tail index. Therefore, the discussion below carries over to dependent sequences, as we focus primarily on $\alpha$.

The limit in (3) explicated in (4) is most easily interpreted by analogy with the central limit theorem. The difference is the focus on order statistics rather than averages, but its usefulness is the same as no detailed knowledge of $F(x)$ is needed to apply the asymptotic theory. A complication is the fact that there are three limit laws, distinguished by the sign of $\gamma$. For reference, a type II limit law obtains when $\gamma>0$, a type III limit law obtains when $\gamma<0$, and a type I limit law holds for $\gamma=0$. In the last case $(1+\gamma x)^{-1 / \gamma}$ is interpreted as $\mathrm{e}^{-x}$. For exchange rate returns the type II limit law is of special interest. We state it here in an often used alternative format that exhibits the tail index $\alpha$ directly: 


$$
\text { Type II: } \begin{aligned}
G_{z}(x) & =0 . & & x \leqq 0, \\
& =\exp \left(-x^{-x}\right), & & x>0,
\end{aligned}
$$

where $\alpha=1 / \gamma$.

Usually economic theory is not informative about the specific distribution $F(x)$ that applies. However, the qualitative characteristics of the economic process may point to the relevant limit law. Consider the following two necessary conditions from De Haan (1976):

Condition 1. If $F \in D($ Type I $G(x))$ and $F(x)<1$ for all $x$, then $\int_{1}^{x} t^{\beta} \mathrm{d} F(t)$ is finite for all $\beta$.

Condition 2. If $F \in D($ Type II $G(x))$, then $F(x)<1$ for all $x$ and $\int_{1}^{x} t^{\beta} \mathrm{d} F(t)$ is finite for $\beta<x$ and infinite for $\beta>\alpha$.

The intuition behind these conditions is as follows. Loosely speaking, the tail of the distribution is either declining exponentially or by a power. In the first case all moments exist, but in the second case the higher moments do not decay rapidly enough when 'weighted' by the tail probabilities to be integrable, i.e. the d.f. $F(x)$ has fat tails. This explains the appearance of the double exponential in the Type I limit law and the exponential format of the Type II as well. The third limit law is characterized by the fact that it has a finite upper endpoint. The tail-fatness of a d.f. $F$ is now suitably defined as the value of the tail index $x$. Anticipating the next section, given that exchange rate returns are strongly fat-tailed and unbounded in principle, the Type II limit law is the relevant one if the maximum yield distribution converges at all.

A sufficient condition on $F(x)$ for the Type II limit to obtain is:

Condition 3. It is sufficient for $F \in D$ (Type II $G(x))$ that it has no finite upper endpoint, and for each $x>0$ and some $\propto>x>0$

$$
\lim _{t \rightarrow \infty} \frac{1-F(t x)}{1-F(t)}=x^{-x}
$$

The latter condition boils down to regular variation at infinity [see Feller (1971, ch. VIII.8) and Mood et al. (1974, p. 261, th. 16)].

The following discussion shows how the above conditions may be employed in specific cases. We return to the d.f.s $F(x)$ that have received most of the attention in the literature on exchange rates. First, the normal and mixtures of the normal possess all moments, and hence Condition 1 
applies. Therefore these alternatives are thin-tailed and seem unfit for modelling exchange rate returns. Second, the Student- $t$ and the sum-stable d.f. both satisfy Condition 2, because not all moments are finite. To verify that the Student-t class satisfies Condition 3 is straightforward [see, for example, Mood et al. (1974, p. 262)]. The sum-stable distribution, which is not to be confused with the max-stable distribution $G(x)$, takes some effort as only in specific cases does a closed-form solution for its density $f_{a}(t)$ exist (i.e. the normal, the Cauchy, and the inverted chi-square, while the so-called characteristic exponent $\alpha$ is respectively 2,1 and $\frac{1}{2}$ ). Ibragimov and Linnik (1971), however, provide the following asymptotic formula for $t$ large and $0<\alpha<2, \alpha \neq 1$ :

$$
f_{x}(t)=\frac{-1}{\pi t} \sum_{n=1}^{\infty} \frac{(-1)^{n}}{n !} \sin \left(\frac{1}{2} n \pi x\right) \Gamma(n x+1) t^{-n x} .
$$

Use this expansion to verify that Condition 3 holds:

$$
\lim _{t \rightarrow \infty} \frac{\sum(n !)^{-1}(-1)^{n} \sin \left(\frac{1}{2} n \pi x\right) \Gamma(n \alpha+1)(t u)^{-n \alpha}}{\sum(n !)^{-1}(-1)^{n} \sin \left(\frac{1}{2} n \pi \alpha\right) \Gamma(n x+1)(t)^{-n x}} \frac{t^{x}}{t^{x}}=u^{-\alpha} .
$$

From this discussion it is immediate that the competing $F(x)$ 's are nested within their limit law $G(x)$, and are distinguished by different values of $\alpha$. Specifically, the leptokurtic sum-stable hypothesis requires $\alpha<2$ and the Student- $t$ class allows for $\alpha \geqq 2$. The idea is now to estimate $x$ directly without a prior commitment to either hypothesis.

Before we turn to the estimation procedures, the case of dependent variates is briefly discussed. Consider, for example, the simple infinite MA process (AR 1 process): $X_{t}=\sum_{j=0}^{\infty} \lambda^{j} \varepsilon_{t-j}$, where the $\varepsilon_{t}$ are i.i.d. sum-stable variates with tail index $\alpha: 1<\alpha<2$, and $0<\lambda<1$. The effect of the dependency is that the larger values have a tendency to come in clusters. This affects the norming constants but not the tail index. Specifically, the limit law in eq. (5) becomes $\exp \left(-\theta x^{-\alpha}\right)$, where $\theta: 0<\theta \leqq 1$, called the extremal index, is a scaling factor that compensates for the clustering. In this example, $\theta=$ $\lambda^{\alpha}\left(1-\lambda^{\alpha}\right)$, [cf. Leadbetter et al. (1983, ch. 3)]. As mentioned in the introduction, the ARCH process is of economic interest as it exhibits both the clustering phenomenon and has a fat-tailed unconditional d.f. Because of the latter fact, Condition 2 is easily seen to hold. Sufficiency is more intricate to obtain. De Haan et al. (1989) have recently proved sufficiency and obtained expressions for the extremal index $\theta$ by means of appropriate mixing conditions.

Broadly speaking, the estimation procedures for $\alpha$ fall into two categories. A traditional approach uses 'yearly maxima' and assumes that each period's maximum exactly follows one of the three limit laws. If the Type II limit law 
applies, direct estimation by maximum likelihood is consistent. However, in this approach time is more or less arbitrarily divided into separate intervals from which one observation is used. As more than one extreme realization may occur in a period, some information could be lost. Another drawback of this method is that the excesses are assumed to follow the limit law exactly, whereas this is only approximately the case. Recently, some estimators have been proposed based on the largest order statistics which require only that the distribution generating these observations is in a sense well behaved. This implies that the remaining estimation error can be solely attributed to the use of finite samples. For example, regular variation at infinity is often a sufficient condition. For this reason the focus is on these methods.

Let $X_{1}, X_{2}, \ldots, X_{n}$ be a sequence of stationary i.i.d. observations from some distribution function $F \in D$ (Type II $G(x)$ ). We are interested in obtaining an estimate for $\gamma$, given that the Type II limit applies. Define $X_{(1)} \leqq X_{(2)} \leqq$ $\cdots \leqq X_{(n)}$ as the ascending order statistics from a sample $X_{1}, X_{2}, \ldots, X_{n}$ of $n$ consecutive exchange rate yields $X_{i}$. The proposed estimator reads:

$$
\left.\hat{\gamma}=\widehat{1 / \alpha}=\frac{1}{m} \sum_{i=1}^{m}\left[\log X_{(n+1, i)}-\log X_{(n-m)}\right)\right]
$$

The statistic $\hat{\gamma}$ first appeared in Hill (1975). Mason (1982) proved that if Condition 3 is satisfied, $\hat{\gamma}$ is a consistent estimator for $\gamma$. By a result of Goldie and Smith (1987), it follows that $(\hat{\gamma}-\gamma) m^{1 / 2}$ is asymptotically normal with mean zero and variance $\gamma^{2}$.

The estimation procedure requires $m(n) \rightarrow \infty$, but for a finite sample it is not known how to choose $m$ optimally. ${ }^{4} \mathrm{~A}$ heuristic procedure is to compute $\hat{\alpha}$ for different $m$ and to select an $m$ in the region over which $\hat{\alpha}$ is more or less constant. There exists such a region because when one uses too few order statistics, then $\alpha$ will vary heavily with $m$ due to inefficiency. In the opposite case the curvature of the distribution $F$ generating the data weighs too heavily, i.e. only the tail probabilities are well approximated by the limit distribution $G$. In the empirical section we conduct a Monte Carlo study to select $m$. Due to the asymptotic normality of $\hat{\gamma}$, the MSE criterion may be used to select an optimal $m$ for given sample size $n$ and d.f.

The implied asymptotic confidence interval allows one to test directly for the two competing hypotheses about $F(x)$, i.e. the sum-stable and Student-t. The former requires $0<\alpha<2$ and the latter allows for $\alpha \geqq 2$. As noted, discrimination between the two hypotheses is hampered by their nonnestedness. However, as our estimate of $\alpha$ is not conditional upon one of the two hypotheses being true, the asymptotic confidence interval may be used to test for $H_{0}: \alpha<2$ against $H_{1}: \alpha \geqq 2$. The asymptotic normality of $\widehat{1 / \alpha}$ may also

this is the case for both the maximum likelihood and order statistics procedures. 
be exploited to compare $\alpha$ estimates from different samples. The following statistic,

$$
Q=\left(\frac{\alpha_{1}}{\hat{\alpha}_{1}}-1\right)^{2} m_{1}+\left(\frac{\alpha_{2}}{\hat{\alpha}_{2}}-1\right)^{2} m_{2},
$$

where the $\alpha$ and $m$ are as in (7) and the subindexes refer to two independent samples, is asymptotically $\chi^{2}(2)$ distributed. It can be used to test for the equality of $\alpha$ over pre-EMS and EMS data, and to test for equality over different exchange rates.

The data we use consist of weekly bilateral exchange rate returns. In view of the third question concerning the time additivity, the returns over extended periods of time are relevant as well. In the case of a sum-stable distribution for weekly returns, it is immediate that monthly, yearly, etc. rates follow the same distribution, as this class of distribution is invariant under addition. For other leptokurtic alternatives like the Student- $t$, this is not the case. What has not been realized in the economics literature, though, is the fact that the tail behavior of alternatives like the Student- $t$ is unaffected by aggregation. That is, $M_{n}$ generated from a Student-t or any finite sum of Student- $t$ variates all tend to follow the Type II limit law, with the same $\alpha$ ! A sufficient condition for this invariance is given in Theorem 2.

Theorem 2. If $1-F(x)$ varies regularly at infinity, i.e. satisfies Condition 3, then the $M_{n}$ from $F(x)$ or any finite convolution of $F(x)$ follow the same limit law.

A lucid proof may be found in Feller (1974, ch. VIII.8). Hence, focusing on the limit law has the advantage that the amount of tail-fatness is invariant to the chosen period length between observations for 'all' fat-tailed alternatives. It guarantees a robustness to our methods that is not present in the others. In the case where the Student- $t$ is estimated from the returns, the frequency of the data may not be immaterial to the amount of leptokurtosis one will find, for example. Unfortunately, a direct test of Theorem 2 does not seem possible due to the interdependency of high and low frequency data. But some testing is possible, as is discussed in the following section.

\section{Empirical results}

The aims of this section are to evaluate the amount of tail-fatness of the bilateral EMS foreign exchange rates. Our data consist of weekly Friday closing quotations of the Belgian franc (BF), French franc (FF), Italian lira (IL), Dutch guilder (DG), British pound (BP), Irish pound (IP), the German mark (GM) and the Danish krone (DK) quoted against the United States 
dollar (US) spot exchange rate from the Harris Bank. The sample covers the period starting $26 \mathrm{March} 1971$ and ending 6 February 1987. The IP/US rate was only available for the period starting 9 March 1979 and ending 6 February 1987, i.e. from the inception date of the EMS onwards. For the period from 10 February 1987 to 23 June 1989, Kempen and Co., Amsterdam, provided daily closing rates for the time aggregation tests. Our motivation for focusing on the EMS rates is that these are likely to generate the most interesting results for all three questions set forth above. The creation of the EMS, for example, constituted a major change in policy, explicitly geared towards affecting the behavior of exchange rates and provides a nice occasion to employ the $Q$ test of eq. (8). The sample is split in a pre-EMS and EMS period, and the tail index estimates for both periods are compared in order to determine whether the formation of the EMS has indeed affected the extreme movements of the exchange rates. Another issue is the continuing debate as to whether the BP should become part of the EMS. The BP data are therefore included in the sample to shed some light on this matter.

As is well known, in an arrangement like the EMS only $n-1$ countries can set their exchange rates independently, whereas the $n$th country has a degree of freedom in choosing its money supply. ${ }^{5}$ In fact, Germany has functioned as the ' $n$th country' of the EMS. Therefore we take the liberty of economizing on the information by presenting evidence for the exchange rates vis-àvis the DM only. The ECU rate used is the ECU-DM rate.

It is well realized why returns rather than levels of exchange rates are the variable of interest for high frequency data. The main reason is that investors compare returns rather than levels, and capital movements are the prime cause of short-run exchange rate fluctuations. The stylized facts of exchange rate data are as follows. The logarithm of the spot rate is non-stationary, whereas returns are stationary. Sample moments of the empirical return distributions indicate the absence of skewness for freely floating convertible currencies, but may be skewed otherwise. The kurtosis usually points to fatness in the tails. The EMS data, when scrutinized by the usual tests, accorded well with the stylized facts. ${ }^{6}$ All rates are significantly leptokurtic, i.e. the kurtosis is significantly different from zero. The weaker currencies, i.e. the BF, FF and IL, tend to be skewed and have non-zero means. This pattern is related to the periods of sustained excessive inflation and official intervention these currencies experienced.

Above we discussed three open issues in the empirical exchange rate distribution literature. To recapitulate, these concern: (1) the type of distribution function and the amount of tail-fatness; (2) parameter stability; and (3)

\footnotetext{
5riangular arbitrage, moreover, implies that the cross rates are not independent.

${ }^{6}$ Details are reported in Koedijk et al. (1989), which is available upon request.
} 
Table 1

Minimizing MSE m-levels. ${ }^{2}$

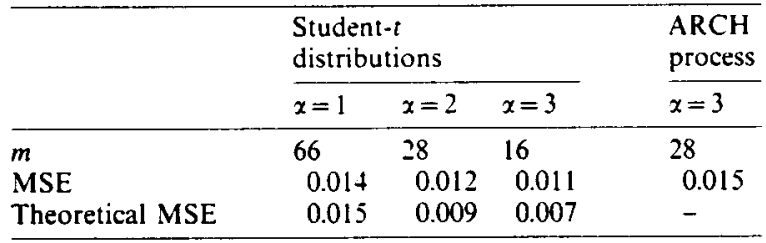

${ }^{2}$ The Monte Carlo experiment consisted of 100 replications of $n=412$ draws from three Student- $t$ distributions and one $\mathrm{ARCH}$ process, after appropriate initialization. The theoretical MSE is computed as $i^{2} / m$ for the i.i.d. cases. Further details are in the main text.

the influence of the data frequency on the tail index. Each of these issues is now addressed empirically on the basis of the EMS data.

We start with the relevancy of the different classes of distribution functions by estimation of the respective tail indices. As noted, the estimators are conditioned upon the $m$ highest order statistics. In order to select appropriate $m$-levels we conducted a Monte Carlo experiment. The $m$-level is selected for which the MSE attains its minimum. The MSE criterion is an appropriate selection criterion given the asymptotic normality of the estimator in eq. (7). We simulated four different distributions, three Student- $t$ distributions with 1, 2, and 3 degrees of freedom, respectively, and a first-order ARCH process with the autoregressive heteroscedasticity parameter equal to 0.73 , which amounts to a tail index of 3 . The values of $\alpha$ were chosen in the range 1-3 with an eye towards the previous studies. Table 1 reports the minimizing $m$-levels and associated MSE. These $m$-levels vary inversely with the true tail index $\alpha$. The reason is that the lower is $\alpha$, the fatter are the tails of the distribution, and hence the more 'outliers' are available for estimation. Above we noted that the MSE is expected to be U-shaped when plotted against $m$, and thus having a global minimum, given the trade-off between efficiency and bias. This was confirmed in all experiments. ${ }^{7}$ From the experiments for the ARCH process it appears that the dependency does not greatly affect the MSE and the choice of $m$. Because of the issue of whether $\alpha>2$ or $x<2$, we choose to condition the estimates on $m=28$. This also accords well with the ARCH experiment.

The tail index estimates are reported in table 2 . From the table it appears that most exchange rates in both periods are characterized by point estimates for $\alpha$ below 2. We can now test formally for $H_{0}: \alpha<2$ against the alternative $H_{1}: \alpha \geqq 2$ on the basis of the asymptotic normality of $\frac{1 / \alpha}{1 / \text { At the }} 5$ percent

${ }^{7}$ Evidence is reported in table A2 in Koedijk et al. (1989). 
Table 2

The tail index. ${ }^{2}$

\begin{tabular}{lll}
\hline & \multicolumn{2}{l}{ Period } \\
\cline { 2 - 3 } & Pre-EMS & EMS \\
\cline { 2 - 3 } & Estimates & \\
\cline { 2 - 3 } Currency & $\dot{\alpha}$ & $\dot{\alpha}$ \\
\hline BF & $2.61(1.64-3.58)$ & $1.49(0.94-2.04)$ \\
FF & $1.92(1.21-2.63)$ & $1.25(0.78-1.71)$ \\
IL & $1.41(0.89-1.93)$ & $1.78(1.12-2.44)$ \\
DG & $1.69(1.06-2.32)$ & $1.62(1.02-2.22)$ \\
BP & $2.66(1.67-3.64)$ & $2.17(1.37-2.98)$ \\
IP & - & $1.63(1.03-2.23)$ \\
DK & $1.59(1.00-2.17)$ & $2.02(1.27-2.77)$ \\
ECU & - & $2.41(1.51-3.29)$ \\
\hline
\end{tabular}

The $95 \%$ asymptotic confidence interval of $x$ is given in parentheses. The estimates are conditioned on $m=28$ and sample size $n=412$.

significance level, $H_{0}$ is never rejected. $H_{1}$ is rejected for the IL over the pre-EMS period, and similarly for the FF during the EMS period. Thus, returns are clearly fat-tailed, i.e. normality or mixtures for that matter are overwhelmingly rejected, but only in some instances can we reject the Student- $t$ with finite variance hypothesis. The sum-stable hypothesis is never rejected. ${ }^{8}$

Next, we enquire into the stability of $\alpha$. Specifically, we ask how the creation of the EMS has affected $\alpha$. A rise in $\alpha$, for example, would implicate a reduction in the extreme volatility of the exchange rate movements. To this end the $Q$ statistic of eq. (8) was calculated for the pre-EMS and EMS period, see table 3 . In four cases, i.e. the BF, FF, DG and BP, the EMS $\hat{\alpha}$ is below the pre-EMS $\hat{\alpha}$, and the reverse holds for the IL and DK. As is apparent from table 3, however, the stability of $\alpha$ cannot be rejected for any of the currencies. In this respect, it seems that the EMS has achieved little to reduce the extreme volatility. This runs counter to popular belief that the EMS has achieved a reduction in uncertainty. Such may still be the case, though, when the behavior in the midsection of the distribution is taken into account. But the shape of the tails of the distribution appear unaffected by the EMS.

The EMS has other goals than reducing the extreme movements. In this respect it is of interest to ask whether the different currencies are sufficiently similar to belong to the same currency area. Similarity may be considered as

\footnotetext{
${ }^{8}$ For the EMS periods the tail indices were also estimated on samples with two weeks centered around each realignment date omitted. The resulting tail estimates, however, were not substantially different from the ones that are presented.
} 
Table 3

Parameter stability.

\begin{tabular}{|c|c|c|c|}
\hline \multirow[b]{4}{*}{ Currency } & \multicolumn{2}{|l|}{ Period } & \multirow[b]{2}{*}{ Interval $^{\mathbf{a}}$} \\
\hline & Pre-EMS & EMS & \\
\hline & \multicolumn{3}{|l|}{ Tail index } \\
\hline & $\dot{x}_{1}$ & $\dot{x}_{2}$ & $x_{1}=x_{2}$ \\
\hline$\overline{\mathrm{BF}}$ & 2.61 & 1.49 & $1.41-2.12$ \\
\hline FF & 1.92 & 1.25 & $1.07-1.82$ \\
\hline IL & 1.41 & 1.78 & $1.07-2.03$ \\
\hline DG & 1.69 & 1.62 & $1.11-2.19$ \\
\hline BP & 2.66 & 2.17 & $1.63-3.11$ \\
\hline DK & 1.59 & 2.02 & $1.22-2.29$ \\
\hline
\end{tabular}

a necessary precondition for achieving such other goals. By using the $Q$ statistic again, we tested for the equality of the tail index between countries over the EMS period. ${ }^{9}$ Equality was never rejected. This suggests that the currencies of the EMS are similar enough to belong to the same currency area, and this conclusion carries over to the BP.

Lastly, we turn to the issue of time aggregation and the use of high and low frequency data. With an eye towards Theorem 2 we computed table 4 . It displays the re-estimated $\alpha$ on the basis of different frequency data for the EMS period. ${ }^{10}$ Evidently, the increased width of the confidence intervals reflects the loss in efficiency due to the reduction in information. No clear rise or fall in the $\hat{\alpha}$ 's can be detected. Quite a number of articles cite a rise in $\alpha$ towards 2 when using lower frequency data. Diebold (1988b) provided other evidence for a tendency towards normality. This may simply be due to the fact that 2 is more likely to be included in the confidence interval when using fewer observations. Unfortunately, one cannot use a statistic like $Q$ to test formally for stability when using different frequency data, as the two samples are not independent.

What may be done, however, is to compare the EMS low frequency estimates with the pre-EMS high frequency estimates. This is done by means of the $Q$ statistic in table 5. Equality is never rejected. We recognize that this constitutes a rather indirect method of testing for equality, but at least it circumvents the dependency problem and generates a confidence interval.

\footnotetext{
${ }^{9}$ The $Q$ statistic may be biased due to triangular arbitrage.

${ }^{10}$ For the different frequency data new $m$-levels were established on the basis of Monte Carlo experiments as before. These results are not presented for considerations of space, but are available from the first author upon request.
} 
Table 4

Estimation with other frequency data. ${ }^{a}$

\begin{tabular}{lllll}
\hline & $\begin{array}{l}\text { Daily } \\
\text { returns } \\
(n=619) \\
(m=30)\end{array}$ & $\begin{array}{l}\text { Bi-weekly } \\
\text { returns } \\
(n=206) \\
(m=13)\end{array}$ & $\begin{array}{l}\text { Tri-weekly } \\
\text { returns } \\
(n=137) \\
(m=11)\end{array}$ & $\begin{array}{l}\text { Monthly } \\
\text { returns } \\
(n=103) \\
(m=10)\end{array}$ \\
\hline BF & 2.56 & 1.71 & 1.54 & 1.60 \\
& $1.64-3.47$ & $0.78-2.63$ & $0.62-2.45$ & $0.61-2.60$ \\
FF & 2.31 & 1.73 & 1.61 & 1.66 \\
& $1.48-3.13$ & $0.79-2.67$ & $0.66-2.56$ & $0.63-2.69$ \\
IL & 2.82 & 1.93 & 2.28 & 2.36 \\
& $1.81-3.83$ & $0.88-2.99$ & $0.93-3.64$ & $0.90-3.82$ \\
DG & 2.93 & 1.68 & 2.20 & 2.10 \\
& $1.88-3.97$ & $0.76-2.59$ & $0.90-3.49$ & $0.80-3.41$ \\
BP & 2.96 & 2.96 & 3.39 & 2.57 \\
& $1.90-4.02$ & $1.35-4.57$ & $1.39-5.39$ & $0.97-4.17$ \\
IP & 2.90 & 1.68 & 1.62 & 1.56 \\
& $1.86-3.22$ & $0.77-2.59$ & $0.66-2.58$ & $0.59-2.53$ \\
DK & 2.37 & 1.28 & 1.78 & 1.91 \\
& $1.52-3.22$ & $0.59-1.98$ & $0.72-2.83$ & $0.72-3.10$ \\
ECU & - & 2.52 & 3.73 & 2.15 \\
& & $1.15-3.89$ & $1.52-5.93$ & $0.82-3.48$ \\
\hline
\end{tabular}

In the first line the estimates of $x$ are presented and the second line gives their $95 \%$ confidence intervats.

The table does not indicate that time aggregation does affect the tail index, i.e. the evidence is in agreement with Theorem 2.

\section{Conclusion}

The paper focuses on three questions concerning exchange rate returns: the amount of tail-fatness, parameter stability, and the effects of time aggregation. As all three are in essence questions about the tail behavior, we have used extreme value theory because it explicitly deals with this. It was found that the main parameter of concern could be estimated directly by simply manipulating some of the higher order statistics. This parameter, the tail index $\alpha$, also characterizes the main alternative classes of distribution functions which have been advanced in the literature. On the basis of the asymptotic confidence interval for $\alpha$, several tests of the hypothesis were performed. Both the direct estimation procedure for the nested models and the confidence intervals constitute an improvement over other methods which have been used previously.

On the basis of the EMS data it was found that the tail index hovers around 2 , and is possibly slightly below 2 , which is weak evidence in favor of the sum-stable hypothesis. In contrast, Boothe and Glassman (1987, pp. 313/314) found evidence in support of the Student-t (with about 3 to 4 degrees 
Table 5

High and low frequency estimates compared.

\begin{tabular}{|c|c|c|c|}
\hline & \multicolumn{3}{|l|}{ Period } \\
\hline & Pre-EMS & EMS (1979-1987) & EMS (1987-1989) \\
\hline & \multicolumn{3}{|l|}{ Frequency } \\
\hline & Weekly returns & Monthly returns & Daily returns \\
\hline & \multicolumn{3}{|l|}{ Tail index } \\
\hline & $\overline{\dot{\alpha}_{1}}$ & $\hat{x}_{2}$ & $\hat{x}_{3}$ \\
\hline $\begin{array}{l}\text { BF } \\
\text { FF } \\
\text { IL } \\
\text { DG } \\
\text { BP } \\
\text { DK }\end{array}$ & $\begin{array}{l}2.61 \\
1.92 \\
1.41 \\
1.69 \\
2.66 \\
1.59\end{array}$ & $\begin{array}{l}1.60 \\
1.66 \\
2.36 \\
2.10 \\
2.57 \\
1.91\end{array}$ & $\begin{array}{l}2.56 \\
2.31 \\
2.82 \\
2.93 \\
2.96 \\
2.37 \\
\end{array}$ \\
\hline \multicolumn{4}{|c|}{ Intervals ${ }^{\mathrm{a}}$} \\
\hline & $x_{1}=x_{2}$ & $x_{2}=x_{3}$ & $x_{3}=x_{1}$ \\
\hline $\begin{array}{l}\text { BF } \\
\text { FF } \\
\text { IL } \\
\text { DG } \\
\text { BP } \\
\text { DK }\end{array}$ & $\begin{array}{l}1.42-2.82 \\
1.12-2.56 \\
0.98-2.05 \\
1.08-2.45 \\
1.59-3.68 \\
1.01-2.30\end{array}$ & $\begin{array}{l}1.43-2.81 \\
1.32-2.80 \\
1.66-3.69 \\
1.67-3.54 \\
1.75-3.93 \\
1.38-3.05\end{array}$ & $\begin{array}{l}1.75-3.41 \\
1.44-2.73 \\
1.64-1.77 \\
1.63-2.41 \\
1.91-3.69 \\
1.36-2.33\end{array}$ \\
\hline
\end{tabular}

of freedom) and the mixture of a normal. The latter hypothesis seems implausible given our $\alpha$ estimates (the mixtures require $\alpha$ to be infinite), but the Student- $t$ with a finite variance (where $\alpha$ corresponds to the degrees of freedom) cannot be ruled out on the basis of our estimates. Exploiting the asymptotic normality of $\widehat{1 / \alpha}$, probably due to the recurrent realignments it was found that the formation of the EMS did little to reduce the extreme risks. On the other hand, equality of the tail indices between the currencies, including the BP, was never rejected. Therefore even if the formation of the EMS did not reduce extreme volatility, the EMS currencies may still be natural companions with gains in other areas. One may want to think about this in terms of the parameters of the underlying d.f. $F(x)$. While the tail index is constant, other parameters determining the center shape of $F(x)$ could have changed. For example, Boothe and Glassman (1987, p. 315) found, admittedly for non-EMS data, that the stability of all parameters of $F(x)$ over time is rejected, but this does not preclude a stable tail index. Finally, no indication was found for other frequency data to affect the $\alpha$ estimates. While an unequivocal comparison between the different frequency estimates is still marred by the non-independent samples problem, our partial 
answer shows that the variations in $\hat{\alpha}$ may well be within the confidence intervals [cf. Boothe and Glassman (1987, p. 308)]. It is hoped that this paper makes clear how extreme value theory may be usefully exploited for some questions in the area of exchange rates, where outliers are the rule rather than the exception.

\section{References}

Boothe, P. and D. Glassman, 1987, The statistical distribution of exchange rates: Empirical evidence and economic implications, Journal of International Economics, 297-320.

Diebold, F.X., 1988a, Testing for bubbles, reflecting barriers and other anomalies, Journal of Economic Dynamics and Control, 63-70.

Diebold, F.X., 1988b, Empirical modeling of exchange rate dynamics (Springer-Verlag, New York).

Engle, R.F., 1982, Autoregressive conditional heteroscedasticity with estimates of the variance of United Kingdom inflations, Econometrica, 987-1007.

Fama, E.F. and R. Roll, 1971, Parameter estimates for symmetric stable distributions, Journal of the American Statistical Association, 331-338.

Feller, W., 1971, An introduction to probability theory and its applications, Vol. 2 (Wiley, New York).

Flood, R.P. and P.M. Garber, 1984, Collapsing exchange rate regimes, Journal of International Economics, 1-13.

Goldie, C.M. and R.L. Smith, 1987, Slow variation with remainder: Theory and applications, Quarterly Journal of Mathematics (Oxford 2nd series) 45-71.

Haan, L. de, 1976, Sample extremes: An elementary introduction, Statistica Neerlandica, $161-172$.

Haan, L. de, S.I. Resnick, H. Rootzén and C.G. de Vries, 1989, Extremal behaviour of solutions to a stochastic difference equation with applications to ARCH-processes, Stochastic Processes and their Applications, 213-224.

Hill, B.M., 1975, A simple general approach to inference about the tail of a distribution, The Annals of Statistics, 1163-1173.

Ibragimov, I.A. and Yu. V. Linnik, 1971, Independent and stationary sequences of random variables, in: J.F.C. Kingman, ed. (Wolters Noordhof, Groningen).

Koedijk, K.G., M.M.A. Schafgans and C.G. de Vries, 1989, The tail index of exchange rate returns, Discussion paper 8908M (Institute for Economic Research, Erasmus Lniversity, Rotterdam).

Leadbetter, M.R., G. Lindgren and H. Rootzen, 1983, Extremes and related properties of random sequences and processes (Springer-Verlag, New York).

Mason, D.M., 1982, Laws of large number for sums of extreme values, The Annals of Probability, 754-764.

McFadden, D., 1973, Conditional logit analysis of qualitative choice behavior, in: P. Zarembka, ed., Frontiers of econometrics (Academic Press, New York).

Mood, A.M., T.A. Graybill and D.C. Boes, 1974, Introduction to the theory of statistics (MuGraw-Hill, New York).

White, H., 1982, Regularity conditions for Cox's test of non-nested hypotheses, Journal of Econometrics, 301-318. 